\title{
Epidemiologia do Abortamento na Adolescência
}

\author{
Epidemiology of Abortion During Adolescence
}

Ariani Impieri Souza ${ }^{1}$, Márcia M. A. Aquino ${ }^{2}$, José Guilherme Cecatti ${ }^{2}$, João Luiz Pinto e Silva²

\begin{abstract}
RESUMO
Objetivo: avaliar o perfil sócio-demográfico e obstétrico das adolescentes internadas por abortamento, comparativamente ao das mulheres adultas internadas pela mesma causa. Material e métodos: Trata-se de um estudo descritivo que avaliou 230 mulheres com complicações por abortamento, das quais 59 eram adolescentes, internadas na Maternidade do IMIP (Instituto Materno-Infantil de Pernambuco) no periodo de agosto de 1994 a julho de 1995. As variáveis estudadas foram: grau de escolaridade, situação marital, atividade remunerada, idade gestacional, número de gestações, desejo de gravidez, uso de MAC, tipo de relacionamento, motivo da interrupção voluntária, classificação clínica do abortamento $e$ complicações associadas. Para a análise dos dados, procedeu-se à distribuição das variáveis entre adolescentes $e$ adultas, utilizando-se os testes estatisticos do $\chi^{2}$ e $\chi^{2}$ para tendência. Resultados: comparativamente à mulheres adultas que abortaram, as adolescentes apresentaram uma menor proporção de atividade remunerada e de multiparidade e uma maior proporção de gestações resultantes de um relacionamento não-estável.

Conclusões: os resultados deste estudo permitem concluir que biologicamente as adolescentes que são internadas por abortamento têm um perfil semelhante ao das mulheres adultas. $O$ que as diferenciam destas são as condições sociais e demográficas desfavoráveis que enfrentam em suas primeiras gestações, geralmente não planejadas.
\end{abstract}

PALAVRAS-CHAVE: Aborto. Adolescência. Mortalidade materna.

\section{Introdução}

A adolescência é a fase de transição entre a infância e a idade adulta. Este período caracterizase por importantes transformações anatômicas (alteração na composição corporal, estirão de crescimento), fisiológicas (maturação sexual, gonadal, esquelética, cardiovascular, respiratória),

\footnotetext{
${ }^{1}$ Instituto Materno-Infantil de Pernambuco (IMIP), Recife, PE

${ }^{2}$ Departamento de Tocoginecologia CAISM/FCM/UNICAMP Correspondência:

José Guilherme Cecatti

Caixa Postal 6030

13083-970 - Campinas - SP

Fax: 019-788.9304
}

mentais (alterações psicológicas) e sociais (responsabilidade e independência). Seus limites são de dificil caracterização e, para a Organização Mundial da Saúde, estende-se dos 10 aos 20 anos incompletos ${ }^{13}$.

A gravidez na adolescência tem se destacado como um problema de saúde pública em vários países do mundo. Nos EUA a cada ano quase um milhão de adolescentes engravidam e, destas, 85\% não são gestações planejadas ${ }^{2}$. As causas da gravidez na adolescência podem ser múltiplas: capacidade reprodutiva precoce, amadurecimento sexual mais acelerado, estilo de vida, casamento tardio e estímulos da mídia para a prática sexual.

A média de idade da menarca nos países desenvolvidos está entre 11 a 12 anos e no Brasil, entre 12 a 13 anos. Esta média está mais baixa do 
que a observada nas décadas passadas, o que favorece a gestação em idade também mais precoce $^{15,16}$.

O início precoce da atividade sexual favorece o aumento da incidência da gestação entre adolescentes. Nos EUA, em 1998, 56\% das mulheres e $73 \%$ dos homens já tiveram a sua primeira relação sexual aos 18 anos $^{2}$. No Brasil, em 1980, o número de mães com até 15 anos no Estado de São Paulo aumentou 30\%, quando comparado ao ano de $1970^{8}$.

As complicações maternas médicoobstétricas da gravidez na adolescência freqüentemente incluem o aborto espontâneo ou provocado, anemia, distócias de parto e a hipertensão gestacional. Destas, sem dúvida, a complicação que mais se associa a danos físicos e psicológicos é o aborto. Além disso, as complicações da gravidez, parto e puerpério constituem a $10^{\mathrm{a}}$ causa de óbitos entre adolescentes brasileiras ${ }^{16}$.

O abortamento é importante causa de mortalidade materna, principalmente nos países onde não é legalizado ${ }^{4,10}$. No Brasil, onde as indicações de aborto legal são restritas, tem sido muito dificil estimar a mortalidade materna por esta causa. Os dados sobre abortamento obtidos no Brasil são baseados fundamentalmente em número de registros de internações por suas complicações e em dados de declarações de óbito $^{1,4,10,14}$.

Na cidade de Recife, o abortamento constitui $11,9 \%$ das causas de óbito materno, após correção de um subregistro de $60 \%$ dos casos, e corresponde à terceira causa de morte materna ${ }^{5}$. Já nos países onde os abortamentos são praticados de forma legal e segura, em geral a morte materna é evento raro e, quando ocorre, está relacionada a complicações anestésicas ${ }^{3,7}$. A taxa de mortalidade materna entre adolescentes americanas com menos de 15 anos é cerca de duas vezes e meia maior do que a encontrada nas mulheres na faixa etária de 20 a 24 anos. No Brasil esta cifra é 30\% maior para as adolescentes quando comparados os mesmos grupos $^{16}$.

O aborto entre adolescentes associa-se a fatores culturais, ao papel social da adolescente, a classe social, aos recursos econômicos e ao acesso a serviços de saúde. O objetivo do presente estudo foi avaliar o perfil sócio-demográfico e obstétrico das adolescentes internadas por abortamento comparativamente ao das mulheres adultas com internação pelo mesmo diagnóstico.

\section{Pacientes e Métodos}

No período de agosto de 1994 a julho de 1995 foram entrevistadas 230 mulheres que procuraram a Maternidade do IMIP (Instituto Materno-Infantil de Pernambuco) em Recife, PE, e que foram internadas para tratamento de complicações de abortamento.

Após a obtenção do consentimento verbal, as mulheres foram entrevistadas na enfermaria, depois de realizados os procedimentos médicos necessários. Utilizou-se questionário com perguntas pré-codificadas e abertas, que posteriormente foram codificadas. O estudo foi previamente aprovado pelo Comitê de Ética da instituição, sendo assegurado o sigilo das informações.

Abortamento foi definido como interrupção da gestação antes da $22^{\mathrm{a}}$ semana de gestação conforme padronização da FIGO - 1976 ${ }^{18}$. As variáveis estudadas foram: grau de escolaridade, situação marital, atividade remunerada da mulher, idade gestacional, número de gestações, desejo de gravidez, uso de método anticoncepcional, tipo de relacionamento, motivo de interrupção da gestação, classificação clínica do abortamento e complicações associadas.

Para a análise dos dados, procedeu-se à descrição comparativa da distribuição percentual das diversas categorias das variáveis estudadas entre adolescentes e adultas. Para a análise da significação estatística, utilizaram-se os testes do $\chi^{2}$ e $\chi^{2}$ para tendência.

\section{Resultados}

Das 230 mulheres com complicações por abortamento, 59 eram adolescentes e 171 tinham 20 anos de idade ou mais. Entre as variáveis sóciodemográficas estudadas, observou-se que não houve diferença estatisticamente significativa entre os grupos em relação à escolaridade da mulher. A distribuição da idade gestacional por ocasião do abortamento foi similar em ambos os grupos (Tabela 1).

Quase 70\% das mulheres com 20 anos ou mais tinham um companheiro, o que ocorreu em cerca de $60 \%$ das adolescentes, diferença esta nãosignificativa. A maioria das adolescentes (quase $80 \%$ ) não exercia atividade remunerada, ao passo que o mesmo ocorria com apenas a metade das mulheres com 20 anos ou mais (Tabela 2). A maior parte das adolescentes $(64,4 \%)$ estava grávida pela primeira vez, ao passo que quase metade das mulheres com 20 anos ou mais cursava pelo menos a $3^{a}$ gestação (Tabela 3).

Observou-se que quase $55 \%$ das adolescentes não usava método anticoncepcional, enquanto que esse valor era de cerca de $43 \%$ para 
as mulheres adultas, o que não foi estatisticamente significativo (Tabela 4). Da mesma forma, não houve diferença na utilização dos diferentes métodos. Também não houve diferença estatisticamente significativa entre as mulheres quanto ao desejo de engravidar. A maioria, mais de $60 \%$, em ambos os grupos, não desejava engravidar.

Quanto ao tipo de relacionamento do qual resultou a gravidez atual, os resultados mostram que havia um número significativamente maior de mulheres adultas cuja gravidez resultou de um relacionamento estável. Cerca de $48 \%$ das

Tabela 1 - Distribuição das mulheres com abortamento por grau de escolaridade e idade gestacional, segundo a idade.

\begin{tabular}{|c|c|c|}
\hline & Até 19 anos & 20 anos ou mais \\
\hline \multicolumn{3}{|l|}{ Escolaridade \# } \\
\hline Sem & 1,7 & 4,2 \\
\hline $1^{\circ} \mathrm{grau}$ incompleto & 57,6 & 43,1 \\
\hline $1^{\circ}$ grau completo & 11,9 & 17,4 \\
\hline $2^{\circ} \mathrm{grau}$ & 28,8 & 29,3 \\
\hline Superior & - & 6,0 \\
\hline $\mathrm{n}$ & 59 & $167^{*}$ \\
\hline \multicolumn{3}{|l|}{ IG (semanas) @ } \\
\hline Até 12 & 72,9 & 65,0 \\
\hline 13 ou mais & 27,1 & 35,0 \\
\hline$n$ & 59 & 171 \\
\hline
\end{tabular}

Tabela 2 - Distribuição das mulheres com abortamento por situação marital e atividade remunerada, segundo a idade.

\begin{tabular}{lccrr}
\hline Condição & até 19anos & 20 anos ou mais & $\chi^{2}$ & \multicolumn{1}{c}{$p$} \\
\hline Solteira/separada/viúva & 39,0 & 30,4 & 1,47 & 0,225 \\
Sematividade remunerada & 79,7 & 50,2 & 14,33 & 0,0001 \\
$\mathrm{n}$ & 59 & 171 & & \\
\hline
\end{tabular}

Tabela 3 - Distribuição das mulheres com abortamento por número de gestações, segundo a idade.

\begin{tabular}{lcc}
\hline$N^{0}$ de gestações & Até 19 anos & 20 anos ou mais \\
\hline Uma & 64,4 & 18,7 \\
Duas & 28,8 & 33,9 \\
3 ou mais & 6,8 & 47,4 \\
$\mathrm{n}$ & 59 & 171 \\
\hline
\end{tabular}

$\chi_{\text {trend }}^{2}=48,55 \quad p<0,00001$ adolescentes tiveram a gravidez resultando de um namoro ou caso, o que ocorreu apenas em cerca de $29 \%$ das adultas (Tabela 5).

Das adolescentes que se internaram por abortamento, $32,2 \%$ relataram ter feito voluntariamente algo para interromper esta gestação, contra $27,5 \%$ das adultas, diferença não-significativa (Tabela 6). Quanto ao motivo da interrupção, a maioria das mulheres adultas $(52,2 \%)$ alegava ser a falta de condições financeiras, o que para as adolescentes foi o segundo principal motivo. Para estas, a falta de preparo foi o motivo mais prevalente (Tabela 6).

Tabela 4 - Distribuição das mulheres com abortamento conforme uso de método anticoncepcional (MAC), segundo a idade.

\begin{tabular}{lcc}
\hline MAC em uso & Até 19 anos & 20 anos ou mais \\
\hline Hormonal & 65,4 & 57,3 \\
Preservativo & 11,5 & 10,4 \\
Natural & 23,1 & 30,2 \\
Outro & - & 2,1 \\
Nenhum & 54,4 & 42,9 \\
$\mathrm{n}$ & $57{ }^{*}$ & 168 ** \\
\hline
\end{tabular}

* Falta informação para 2 mulheres

** Falta informação para 3 mulheres

$\chi^{2}=3,30 \quad p=0,508 \quad$ Nenhum $x$ qualquer: $\chi^{2}=2,28 \quad p=0,131$

Tabela 5 - Distribuição das mulheres com abortamento por tipo de relacionamento, segundo a idade.

\begin{tabular}{lcc}
\hline Tipo de relacionamento & Até 19 anos & 20 anos ou mais \\
\hline Namoro/caso & 47,4 & 29,2 \\
Casamento & 11,9 & 24,0 \\
Coabitação & 39,0 & 46,8 \\
Estupro & 1,7 & - \\
$\mathrm{n}$ & 59 & 171 \\
\hline
\end{tabular}

$\chi^{2}=10,87 \quad p=0,012$

Tabela 6 - Distribuição das mulheres com abortamento pelo motivo da interrupção da gestação, segundo a idade.

\begin{tabular}{lcc}
\hline Motivo & até 19 anos & 20 anos ou mais \\
\hline Falta de condições financeiras & 26,3 & 52,2 \\
Não estava preparada & 31,6 & 13,0 \\
Parceiro não queria & 21,0 & 10,9 \\
Família não aceitava & 5,3 & 4,3 \\
Era solteira & - & 2,2 \\
Outros & 15,8 & 17,4 \\
Nada fez para interromper & 67,8 & 72,5 \\
$\mathrm{n}$ & 59 & 170 * \\
\hline
\end{tabular}

* Falta informação para 1 mulher

$\chi^{2}=7,04 \quad p=0,317 \quad$ Nada $x$ algo: $\chi^{2}=0,27 \quad p=0,60$ 
Não houve diferença estatisticamente significativa entre os grupos quanto à classificação clínica do abortamento (incompleto, retido, infectado e inevitável). O mesmo ocorreu quando as mulheres foram analisadas segundo algumas complicações do quadro de abortamento (Tabela 7).

Tabela 7 - Distribuição das mulheres com abortamento conforme a presença de algumas complicações, segundo a idade.

\begin{tabular}{lcccc}
\hline Complicações & até 19 anos & 20 anos ou mais & $\chi 2$ & $\mathrm{p}$ \\
\hline Hemorragias & 91,5 & 94,7 & 0,79 & 0,373 \\
Infecção & 5,1 & 7,6 & Fisher & 0,767 \\
Perfuração & - & 1,2 & Fisher & 1,000 \\
Necessidade de transfusão & 1,7 & 2,3 & Fisher & 1,000 \\
Necessidade de antibióticos & 11,9 & 7,6 & 0,54 & 0,463 \\
\hline
\end{tabular}

\section{Discussão}

A análise dos resultados deste estudo permite notar o que diferencia o perfil sóciodemográfico e obstétrico da adolescente em relação à mulher adulta que aborta e é internada por qualquer complicação relacionada. As adolescentes encontram-se, na maioria das vezes, sem atividade remunerada, têm companheiro fixo menos freqüentemente, estão grávidas pela primeira vez, muitas delas engravidam como resultado de um relacionamento não-estável e interrompem a gravidez por não se acharem preparadas para assumir a maternidade.

No entanto, os grupos são comparáveis quanto à escolaridade, ao uso de métodos anticoncepcionais, ao desejo de engravidar e ao fato de assumir ter feito algo para interromper a gestação. Também são semelhantes quanto à classificação clínica de abortamento e às complicações. Esta semelhança entre os grupos tem sido também mostrada por estudos caso-controle, já que é muito dificil avaliar a idade como um fator de risco para complicações do abortamento, sem analisar a influência de outros. Estudo caso-controle realizado com a mesma casuística, para identificar fatores associados à hospitalização por abortamento, não associou a adolescência como um fator de risco; houve inclusive uma tendência estatisticamente significativa de aumento do risco com o aumento da idade ${ }^{17}$.

Se a adolescente que engravida é aquela que na maioria das vezes está namorando, não está inserida no mercado de trabalho e não está usando método anticoncepcional, parece lógico pensar que falta orientação sobre o planejamento familiar para estas jovens. No entanto, o enfoque não é tão simples. Fornecer orientações sobre métodos anticoncepcionais para adolescentes envolve a participação de múltiplos profissionais, intensa sensibilização e a necessidade de discussão com as próprias adolescentes sobre sua oportunidade, para evitar intervenções e programas não-eficientes.

A discussão sobre métodos mais apropriados é fundamental, já que se sabe que a motivação para o uso de qualquer deles é um dos fatores que mais influencia em sua adesão. A inclusão dos parceiros para esta discussão é outro ponto chave para a boa utilização do método escolhido.

A maioria das mulheres, adolescentes ou não, estava usando um método considerado seguro (hormonal), o que induz à suposição de que estes métodos falharam. Porém, a inadequada utilização do método pode ter influenciado os resultados. Vale a pena salientar que o preservativo, outro método seguro quando bem utilizado, foi usado em segundo lugar como contraceptivo. $\mathrm{O}$ acesso ao planejamento familiar tem sido referido como uma das soluções para diminuir a mortalidade materna por complicações de abortamento ${ }^{6}$. Também se recomenda a necessidade de envolver a participação do homem nos programas de planejamento familiar, tarefa especialmente dificil no caso dos adolescentes.

A melhoria na qualidade da assistência hospitalar, tanto técnica quanto humana, prestada às mulheres que necessitam de atendimento por causa de abortamento é outra medida que tem sido sugerida para diminuir as conseqüências negativas do abortamento, assim como a revisão e adequação das leis a respeito do abortamento ${ }^{9,12}$.

Neste estudo, o principal motivo que levou as adolescentes a interromperem a gestação foi o sentimento de falta de preparo para a maternidade. Da mesma forma, um estudo americano mostrou que as principais razões que levam as jovens a interromperem sua gestação foram, pela ordem, a mudança que um filho poderia acarretar em suas vidas, o sentimento de falta de preparo e a falta de condições financeiras ${ }^{2}$.

Considerando as repercussões fisicas de um abortamento induzido sem assistência médica e sabendo-se que o agravo do ponto de vista social e psicológico é ainda maior nesta faixa etária, muitos estudos insistem na necessidade de rever a legislação a respeito. Um estudo americano que aplicou um questionário para avaliar a atitude, com relação ao abortamento em adolescentes, de médicos pediatras ou com especialização em adolescentes, mostrou que $96 \%$ prescreveriam anticoncepção para elas e $61 \%$ identificariam o aborto como uma opção para gestantes adolescentes, em qualquer circunstância ${ }^{11}$.

Vários autores têm mostrado que a freqüência e a gravidade das complicações do abortamento induzido têm diminuído na última década e tal ocorrência deve-se à menor utilização de métodos inseguros devido ao surgimento do misoprostol. Este análogo da prostaglandina $\mathrm{E}_{1}$ 
popularizou-se e tem sido largamente utilizado pelas mulheres, mesmo em países onde seu uso não é liberado para tal finalidade ${ }^{17}$. O presente estudo, da mesma forma, também mostrou que complicações mais graves como infecção e perfuração apresentaram baixa prevalência, de maneira similar em ambos os grupos.

Finalizando, este estudo possibilita concluir que biologicamente as adolescentes que são internadas por abortamento têm perfil semelhante ao das mulheres adultas, diferenciando-se com relação às condições sociais desfavoráveis que enfrentam em suas primeiras gestações, geralmente não-planejadas. Para diminuir o impacto físico, psicológico e social da gestação, do abortamento induzido sem assistência médica e da parturição entre as adolescentes, é necessária a melhoria no acesso ao planejamento familiar para ela e seu parceiro, na qualidade da assistência hospitalar e discussão mais ampla sobre as leis que regem a interrupção da gestação.

\section{SUMMARY}

Purpose: to evaluate the social, demographic and obstetrical profile of adolescents as compared with adult women hospitalized for abortion complications.

Material and methods: this is a descriptive study that evaluated 230 women with abortion complications. Among them, 59 were adolescents hospitalized at the IMIP Maternity (Recife, Brazil) from August 1994 to July 1995. The variables studied were: educational level, marital status, any paid activity, gestation age, number of pregnancies, desire to become pregnant, use of anticonceptive method, kind of relationship, reason for voluntary interruption, clinical classification of abortion and associated complications. The procedure for data analysis was the distribution of variables among adolescents and adults, the differences being evaluated through $\chi^{2}$ and $\chi^{2}$ for trend.

Results: compared with the adult women who aborted, the adolescents showed a lower number of paid activity and multiparity and a higher number of pregnancies resulting from an unstable relationship.

Conclusions: the results indicated that biologically the adolescents who were hospitalized for abortion have a similar profile to adult women. What differentiates them are the unfavorable social and demographic conditions that they are faced with at their generally unplanned first pregnancies.

KEY WORDS: Abortion. Adolescence. Morbidity.

\section{Referências}

1. Alan Guttmacher Institute. Aborto clandestino: uma realidade latino-americana. New York; 1994.
2. Alan Guttmacher Institute. Teen sex and pregnancy. New York; 1998.

3. Atrash HK, Cheek TG, Hogne CJ. Legal abortion mortality and general anesthesia. Am J Obstet Gynecol 1988; 158: 420-4.

4. Braga LFCO, Soares VMN, Carvalho MTW, Paiva, MP, Nazareno ER, Hirati VM, et al. Relatório dos comitês de morte materna do Paraná - 1992. Femina 1994; 22: 481-92.

5. Cecatti JG, Albuquerque RM, Hardy E, Faúndes A. Mortalidade materna em Recife. Causas de óbitos maternos. Rev Bras Ginecol Obstet 1998; 20: 7-11.

6. Faúndes A, Hardy H, Cecatti JG. Planejamento familiar e saúde materno infantil. Femina 1991; 19: 189-98.

7. Frank P, McNamee R, Hannaford PC, Kay CR, Hirsch $\mathrm{S}$. The effect of induced abortion on subsequent fertility. Br J Obst Gynaecol 1993; 100: 575-80.

8. Henriques MH, Silva NV, Singh S. Adolescentes de hoje, país do amanhã: Brasil. New York: Allan Guttmacher Institute; 1989.

9. Kohl SG, Lanman JT. Further observations on changes in pregnancy outcome following liberalization of the New York State abort law. Am J Obstet Gynecol 1974; 118: 485-92.

10. Laguardia KD, Rotholz MV, Belfort P. A 10-year review of maternal mortality in a municipal hospital in Rio de Janeiro: a cause for concern. Obstet Gynecol 1990; 75: 27-32.

11. Miller NH, Miller DJ, Pinkston Koenigs LM. Attitudes of the physician membership of the Society for Adolescent Medicine toward medical abortion for adolescents. Pediatrics 1998; 101: E4.

12. Misago C. Preventing unsafe abortion and limiting its consequences. Cohort can be done? Kangoroo 1994; 2: 172-7.

13. Organización Mundial de La Salud - El embarazo y el aborto en la adolecencia. 1975 Ginebra: OMS.

14. Organización Mundial de La Salud - Aborto provocado. Informe de um grupo cientifico de la OMS. 1978 Ginebra: OMS.

15. Pinto e Silva JLC. Aspectos biológicos e sociais da gravidez na adolescência. J Bras Ginecol 1984; 94: 227-32.

16. Rodrigues AP, Souza MCB, Brasil RMC, Carakushansky G. Gravidez na adolescência. Femina 1993; 21: 199-223.

17. Souza AI, Cecatti JG, Ferreira LOC, Santos LC. Risk factors for hospitalization due to abortion at IMIP, Recife, Brazil.. J Trop Ped In press.

18. WHO: Recomended definitions, terminology and format for statistical tables related to the perinatal period and use of a new certificate for cause of perinatal deaths: modifications recommended by FIGO as amended. October 14, 1976. Acta Obstet Gynecol Scand 1977; 56: 247-53. 